\title{
El tamizaje poblacional del cáncer de ovario no redujo la mortalidad por esta enfermedad en el seguimiento a largo plazo
}

\author{
Population-based screening for ovarian cancer did not reduce mortality from this disease in long-term follow-up
}

\author{
Comentado de: \\ Menon U, et al. Lancet. 2021;397(10290):2182-2193. PMID: \\ $33991479^{1}$
}

\section{Objetivos}

Evaluar la mortalidad a largo plazo asociada al cáncer de ovario y trompas de Falopio en mujeres posmenopáusicas sometidas a dos estrategias de tamizaje distintas. Como objetivo secundario, evaluar la incidencia y el estadio de estas enfermedades según las distintas estrategias de tamizaje.

\section{Diseño y lugar}

Ensayo clínico controlado y aleatorizado realizado en Gran Bretaña, conocido como UKCTOCS (UK Collaborative Trial of Ovarian Cancer Screening).

\section{Participantes}

Se reclutaron mujeres posmenopáusicas de 50 a 74 años de 13 centros del Servicio Nacional de Salud en Inglaterra, Gales e Irlanda del Norte. Los criterios de exclusión fueron la ooforectomía bilateral, las neoplasias malignas de ovario o no ováricas activas, o el riesgo aumentado de cáncer de ovario familiar.

\section{Intervención}

Entre 2001 y 2005, un total de 202.638 mujeres posmenopáusicas fueron asignadas al azar, en una proporción de 2:1:1, utilizando un algoritmo de números aleatorios generado por computadora, a recibir: a) ninguna intervención de tamizaje $(n=101.314)$; b) tamizaje anual con determinación de CA125 (interpretado utilizando un algoritmo de predicción de riesgo de cáncer de ovario, basado en las variaciones en el tiempo del CA 125, ROCA $\left.{ }^{\circledR}\right)^{2}$, con ecografía transvaginal como prueba de segunda línea (tamizaje multimodal; $\mathrm{n}=50.640$ ); o c) detección anual con ecografía transvaginal sola (ultrasonido, $n=50.639$ ) en una proporción de 2:1:1 utilizando un algoritmo de números aleatorios generado por computadora.

El tamizaje terminó en 2011, y el seguimiento de las participantes (mediana de 16 años; intervalo intercuartilo 15,1 a 17,3), en junio de 2020.

\section{Resultados}

De las 202.562 mujeres incluidas en el análisis, 2.055 fueron diagnosticadas con cáncer de ovario o de las trompas, $522 / 50.625(1 \%)$ de las participantes en la rama de tamizaje multimodal, 517/50.623 (1\%) en la rama ultrasonido, y 1.016/101.314
( $1 \%$ ) en la rama no tamizaje. Sin embargo, no se observó diferencia en la mortalidad por esta enfermedad en las comparaciones realizadas: $296 / 50.625(0,6 \%)$ en la rama de tamizaje multimodal, $291 / 50.623(0,6 \%)$ en la rama ultrasonido, y 619/101.314 (0,6\%) en la rama no tamizaje. No se observó una reducción significativa en las muertes por cáncer de ovario y de trompas en los grupos de tamizaje multimodal $(p=0,58)$ o ultrasonido $(p=0,36)$ en comparación con el grupo sin detección.

Por otro lado, la modalidad multimodal de tamizaje mostró un aumento absoluto del $13 \%$ en la incidencia de la enfermedad en estadio I y II, en relación tanto a la ecografía como al no tamizaje (la rama ultrasonido no mostró diferencias en la distribución por estadio). Este aumento del diagnóstico de cáncer de ovario o de la trompa en estadios iniciales en la rama de tamizaje multimodal fue fundamentalmente a expensas de la disminución de la incidencia de la enfermedad en estadio IV, pero no hubo cambios en el diagnóstico en estadio III (enfermedad intraabdominal), con una relación estadio III:IV de 2:1.

\section{Conclusión}

El seguimiento a largo plazo (seguimiento medio mayor a 16 años después del reclutamiento) en el mayor ensayo de detección de cáncer de ovario y de las trompas proporciona evidencia definitiva de que ninguno de los enfoques de tamizaje utilizados en UKCTOCS redujo las muertes por cáncer de ovario, en comparación con ninguna prueba de tamizaje, a pesar de la disminución del $10,2 \%$ en la incidencia general de la enfermedad en estadio III o IV. Por este motivo, no se puede recomendar ninguna estrategia de tamizaje de esta enfermedad a la población general.

Fuentes de financiamiento/Conflicto de interés de los autores: Estudio financiado por el Instituto Nacional para la Investigación en Salud de Reino Unido y las organizaciones sin fines de lucro Cancer Research UK y The Eve Appeal. Algunos de los autores declararon tener acciones en Abcodia, empresa que tiene la licencia de ROCA desde 2014, recibir regalías y honorarios de esa empresa, mientras que otros fueron co-inventores de este algoritmo y co-propietarios de la patente (originalmente otorgada a Massachusetts General Hospital y Queen Mary University of London, expirada en la actualidad). También fueron declaradas por los autores la tenencia de una patente para el diagnóstico del cáncer de mama, opciones de acciones de SISCAPA Assay Technologies, haber recibido subsidios de instituciones gubernamentales y organizaciones sin fines de lucro durante el presente estudio, y/o de empresas privadas fuera del mismo.

\section{Comentario}

Una de las fortalezas de este estudio, además de haber sostenido el seguimiento de más de 200.000 pacientes por 20 años, radica en que el desenlace principal fue la mortalidad por cáncer de ovario, el verdadero indicador de impacto de una intervención en población sana. Los resultados informados sugieren que la mejoría diagnóstica (aumento de la incidencia de cánceres en estadios iniciales) no se tradujo en una mejoría pronóstica. El aumento observado de diagnósticos en estadios iniciales no fue atribuido por los autores a sobrediagnóstico, ya que la incidencia de la enfermedad fue similar en las tres ramas durante los 20 años de seguimiento. De hecho, en la rama de tamizaje multimodal, los resultados oncológicos de los estadios I y II fueron peores que en la rama ecografía o ninguna intervención (la mortalidad por cáncer de ovario estadio 
I en esa rama fue $24 \%$ frente a $13 \%$ en la rama ecografía y $12 \%$ en la rama no tamizaje). Además, los autores analizaron y desestimaron la posibilidad de dilución del efecto del tamizaje por el largo seguimiento luego de la finalización de la intervención (2011 a 2020), con similares cocientes de riesgo de mortalidad a los 15 y 18 años.

El grupo de trabajo de este estudio comenzó en la década de 1990 analizando la variabilidad del CA 125 a lo largo del tiempo como predictor del cáncer de ovario ${ }^{3}$. Además de haber desarrollado un programa de algoritmo diagnóstico, denominado ROCA, tienen participación en la compañía propietaria. Esto implica que los autores estaban muy interesados en demostrar el impacto de realizar las pruebas de tamizaje en la disminución de la mortalidad por esta enfermedad. El trabajo mostró que efectivamente, el abordaje multimodal aumentaba el diagnóstico inicial de tumores biológicamente agresivos, pero no lo suficiente como para cambiar la historia natural de la enfermedad.

Por estas latitudes no tenemos ROCA, tenemos ecografía transvaginal. En este contexto, el ensayo UKCTOCS replica lo ya observado en otro conocido ensayo clínico de rastreo de cáncer de pulmón, próstata, ovario y colon (PLCO, por sus iniciales en inglés $)^{4}$ : hacer tamizaje anual con ecografía transvaginal en mujeres postmenopáusicas no ha demostrado disminuir la mortalidad asociada al cáncer de ovario. Esto reafirma las recomendaciones ya realizadas por la Fuerza de Tareas de Servicios Preventivos de los EE.UU. y la Academia Americana de Médicos de Familia que expiden su recomendación en contra de la realización de tamizaje de cáncer de ovario en mujeres asintomáticas ${ }^{5,6}$.

En la rama ultrasonido, el número de cirugías por cada cáncer de ovario invasor detectado fue de 35. Si este impactante número se da en el marco de un ECCA, en el que el manejo está protocolizado, definido por criterios clínicos y ecográficos, cabe preguntarse, en nuestro perverso sistema sanitario, ¿cuántas mujeres postmenopáusicas serán operadas por la solicitud inapropiada de la ecografía transvaginal?

\section{Conclusiones de la comentadora}

No hay hasta el momento estrategia de tamizaje para el cáncer de las trompas y los ovarios que haya demostrado beneficio. Por este motivo, ante la ausencia de beneficio, no se debe solicitar ecografía transvaginal a mujeres posmenopáusicas asintomáticas con riesgo habitual de desarrollar esta enfermedad.

Marina Lamm [ Servicio de Ginecología, Hospital Italiano de Buenos Aires. marina.lamm@hospitalitaliano.org.ar ]

Lamm M. El tamizaje poblacional del cáncer de ovario no redujo la mortalidad por esta enfermedad en el seguimiento a largo plazo. Evid Actual Pract Ambul. 2021;24(3):e002136. Available from: https://dx.doi.org/10.51987/EVIDENCIA.V24l4.6959. Comentado de: Menon U, et al. Ovarian cancer population screening and mortality after long-term follow-up in the UK Collaborative Trial of Ovarian Cancer Screening (UKCTOCS): a randomised controlled trial. Lancet. 2021 Jun 5;397(10290):2182-2193. PMID: 33991479

\section{Referencias}

1. Menon U, Gentry-Maharaj A, Burnell M, et al. Ovarian cancer population screening and mortality after long-term follow-up in the UK Collaborative Trial of Ovarian Cancer Screening (UKCTOCS): a randomised controlled trial. Lancet. 2021;397(10290):2182-2193. Available from: 10.1016/s01406736(21)00731-5;https://dx.doi.org/10.1016/s0140-6736(21)00731-5.

2. The ROCA ${ }^{\circledR}$ Test for Ovarian Cancer;. Available from: https://www.therocatest.co.uk/ [Last access: 2021-07-21].

3. Jacobs IJ, Skates SJ, MacDonald N, et al. Screening for ovarian cancer: a pilot randomised controlled trial. Lancet. 1999;353(9160):1207-1210. Available from: 10.1016/S0140-6736(98)10261-1.

4. Buys SS, Partridge E, Greene MH, et al. Buys et al Ovarian cancer screening in the Prostate, Lung, Colorectal and Ovarian (PLCO) cancer screening trial: findings from the initial screen of a randomized trial. Am J Obstet Gynecol. 2005;193(5):1630-1639. Available from: 10.1016/j.ajog.2005.05.005.

5. US Preventive Services Task Force. Screening for Ovarian Cancer: US Preventive Services Task Force Recommendation Statement. JAMA. 2018;319(6):588-594. Available from: 10.1001/jama.2017.21926.

6. ; 2021. Available from: https://www.aafp.org/family-physician/patient-care/clinical-recommendations/all-clinical-recommendations/ovarian-cancer. html [Last access: 2021-07-23]. 